\title{
Static GPU Threads and an Improved Scan Algorithm
}

\author{
Jens Breitbart \\ Research Group Programming Languages / Methodologies \\ Universität Kassel \\ Kassel, Germany \\ jbreitbart@uni-kassel.de
}

\begin{abstract}
Current GPU programming systems automatically distribute the work on all GPU processors based on a set of fixed assumptions, e.g. that all tasks are independent from each other. We show that automatic distribution limits algorithmic design, and demonstrate that manual work distribution hardly adds any overhead. Our Scan ${ }^{+}$algorithm is an improved scan relying on manual work distribution. It uses global barriers and task interleaving to provides almost twice the performance of Apple's reference implementation [1.
\end{abstract}

\section{Introduction}

Graphics processing units (GPUs) are a compelling platform for High Performance Computing (HPC) as they offer multiple times the processing power and memory bandwidth of modern CPUs. However, GPUs are a rather new HPC platform and their programming systems are still at an early stage of its development. NVIDIAs CUDA and the Open Compute Language (OpenCL) are the most modern systems currently available, yet they limit algorithmic design with a fixed work distribution scheme and the lack of global synchronization.

Current GPUs are tiled many-core systems, consisting of tiles of closely coupled processors. We refer to these tiles as multiprocessing elements (MPEs). In both CUDA and OpenCL, developers must define as many tasks as possible. These tasks are automatically mapped on the MPEs, which allows for transparent scalability, but as the mapping order of the tasks is undefined, one cannot use intertask synchronization. However, intertask synchronization and manual mapping of tasks to MPEs is a requirement for complex algorithm. We introduce the notion of static workgroups, which are similar to CPU threads in the sense that they process not one task but multiple tasks sequentially. Static workgroups are implemented by fully occupying the GPU with workgroups and manually map the tasks to these static workgroups. Using static workgroups in scenarios were they are of no algorithmic benefit decreases performance slightly, since the automatic scheduling is more efficient than our manual scheduling. However, control over task mapping allows for easy sharing of data between tasks mapped to the same workgroup, as data can be kept in fast on-chip memory. For example it 
allows sharing data between matrix multiplication tiles and thereby prevents reoccurring reads from slow off-chip memory.

Relying on manual work distribution and global synchronization allows for implementing an improved scan (prefix sums) algorithm. We refer to this algorithm as Scan ${ }^{+}$. The algorithm is based on the algorithm by Blelloch [2, which was e.g. implemented by Harris et al. 5] for GPUs. Scan ${ }^{+}$removes the need for multiple kernel calls by using global synchronization and interleaves the two phases of the scan algorithm to allow for higher processor utilization. These techniques allow a performance increase of about a factor of two.

We experienced with OpenCL and CUDA and measured the performance of our implementation with a GeForce GTX 280.

The paper is organized as follows. First, Sect. 2 gives a basic introduction in GPU architecture and Sect. 3 explains the CUDA and OpenCL programming model. The next section introduces our concept of static workgroups and shows its performance with a basic matrix multiplication. We show both the overall performance decrease compared to a standard CUDA implementation with automatic work distribution and the speedup to demonstrate that manual work distribution allows for good scalability. Section [5] describes the $\mathrm{Scan}^{+}$algorithm and its benefits compared to the well known algorithm. The paper finishes with related work and a conclusion in Sects. 6] and [7, respectively.

\section{GPU Architecture}

GPUs are considered to be one of the first many-core architectures with hundreds of slow in-order cores not providing decent single thread performance. The cores are organized in tiles, meaning subsets of the processors are bundled together. These architectures allow for fast communication and synchronization within the tile, but communication between processors of different tiles is rather slow. On current GPUs, communication between tiles must be done using off-chip memory. In NVIDIA hardware these tiles are called streaming multiprocessors. We continue to refer to these tiles as MPEs and call a single processor a processing element (PE). On GPUs, PEs are oversatured with threads and the hardware uses this oversaturation to hide memory latency. The number of threads that can be executed at once depends on a set of factors, e.g. the number of registers required by a thread and the number of registers available in the hardware. Detailed rules can for example be found in 4. The hardware used for our measurements has $30 \mathrm{MPEs}$ with $8 \mathrm{PE}$ each.

\section{OpenCL/CUDA Programming Model}

In this section we give a brief introduction to the OpenCL programming model. CUDA's programming model is almost identical to that of OpenCL and mostly differs by names. We use the OpenCL names throughout the following sections, even for programs written in CUDA. In OpenCL the GPU (called compute device) is exposed to the CPU (called host) as a co-processor with its own memory 
subsystem. The host launches special functions on the device called kernels, which are similar to main functions in CPU programs.

Kernel execution is done in an SPMD fashion with normally up to hundreds of thousands of microtasks (called workitems in OpenCL). Microtasks must be bundled together in (data parallel) tasks called workgroups. All workitems in one of these groups can be synchronized and share fast on-chip memory called local memory. A set of workgroups is scheduled to a MPE, which than executes these groups in parallel. How many workgroups a MPE can execute in parallel depends on the hardware and the kernel, as described before. If there are more groups than the hardware can execute in parallel, an external queue is generated and a group from the queue is executed after another one has completed execution. The order in which the workgroups are scheduled is undefined. User can therefore e. g. not have one workgroup wait for completion of another one, since this can result in a deadlock. Furthermore, a global barrier is not possible, since it is not guaranteed that all workgroups are currently active.

In the following section we enhance the programming model to give users access to the scheduling of the workgroups and also allow for global barrier synchronization.

\section{Static GPU Threads}

In OpenCL the mapping of tasks (workgroups) to the hardware is intransparent to the user. We now introduce so called static workgroup or MPE threads that compute not only one task but multiple - static workgroups are therefore similar to CPU threads. To implement static workgroups one must identify how many workgroups can be executed at the device at once and than only start this amount of workgroups, so the device is fully utilized and no workgroup is in the external queue. Unfortuatly OpenCL does not allow this to be queried, so the developer must rely on hardware dependent code calculating this number.

One may implement different scheduling schemes to distribute the tasks to the static workgroup, for example a static scheduling can be implemented with a loop, which indexes depend on the (static) workgroup index. However, one may also decide to implement forms of dynamic scheduling using an atomically incremented counter. Without static workgroups, both CUDA and OpenCL do not allow users to specify the work scheduling and expect tasks all of the same size or it may result in poor performance. Furthermore static workgroups allow for synchronization of workitems in different blocks, so it is e. g. possible to have a global barrier for all workitems, since all workitems are active at the same time.

However as the current programming systems do not natively support static workgroups, using them results in more complex programs, as even static scheduling requires an additional loop. More complex kernel may increase the register pressure, which can decrease overall performance. Figure 1 shows matrix multiplication performance implemented in CUDA. The non-optimized version runs the well known blocked matrix multiplication algorithm that is also being used in NVIDIA's CUDA SDK sample. We can see that the implementation using static 


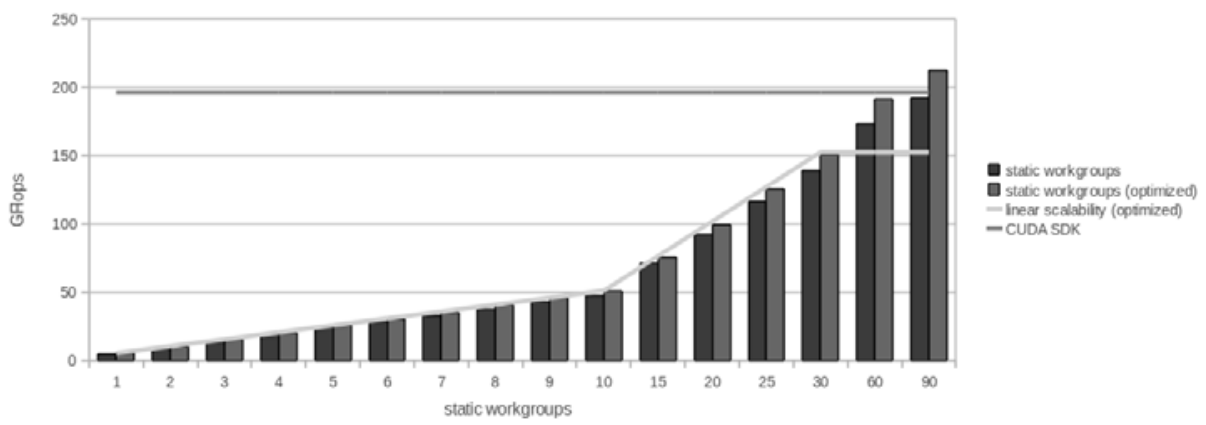

Fig. 1. GTX 280 Matrix multiplication performance (matrix sizes $4800 * 7200 * 4800$ )

threadblocks scales linearly up to the 30 MPEs and oversaturation the MPEs increased performance as well. The overall performance is slightly below that of the SDK implementation. This is the result of the overhead being added by using static threadblocks, however as we can see the overhead is rather small. We have implemented an optimized version that shares data in local memory between tasks. This version calculates not one tile with one static workgroup at a time, but interleaves the computation of two tiles and thereby can utilize the shared memory more efficiently. See figure 2 for an example. The blocks that are in the same row in the result matrix $C$ require the same data from $A$, so interleaving reduces the amount of data that must be fetched from off-chip memory.

A

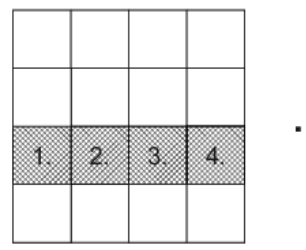

B

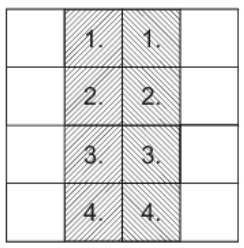

C

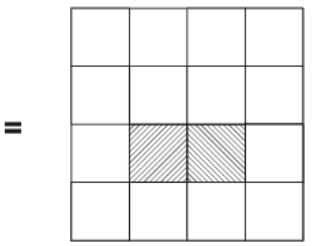

Fig. 2. Optimized blocked matrix multiplication

\section{$5 \mathrm{Scan}^{+}$Algorithm}

In this section we first introduce the CUDA parallel prefix sums implementation suggested by Harris et al. [5] and afterwards outline a way of how this algorithm can be improved using static workgroups. We end this section with a performance comparison to Appel's OpenCL implementation of Harris work.

All prefix sums - also known as scan - is an important parallel building block used in a wide variety of algorithms. Scan takes an input array $\left[x_{0}, x_{1}, \ldots, x_{n-1}\right]$ 
and a binary associative operator + with the identity $I$ as input and returns $\left[I, x_{0},\left(x_{0}+x_{1}\right), \ldots,\left(x_{0}+x_{1}+\ldots x_{n-2}\right)\right]$. Harris et al. suggested a work-efficient implementation for CUDA, which subdivides the whole array into blocks and uses workgroups to perform a local reduction in every block.

The local reduction is split into two phases: first the up-sweep phase and afterwards the down-sweep phase. The array access pattern in both phases is based on a balanced tree. During the up-sweep phase the tree is traversed from the leaves to the root computing partial sums. In the down-sweep phase the tree is traversed from the root to the leaves using the partial sums to compute the missing elements. Figures 3 and 4 show the algorithm in detail. After the up-sweep, the block sums of every workgroup are written to an auxiliary array (SUM). In a second kernel, SUM is scanned and in a third kernel, the result of the scan are used to update the originally array, so it contains the final result. We identify two major performance problems:

1. This algorithm requires 3 kernel calls in the best case. In case more than 1024 workgroups are used, we must recursively apply the algorithm to scan the SUM array. This complicates implementation by a great deal and every kernel call also imposes overhead to the CPU.

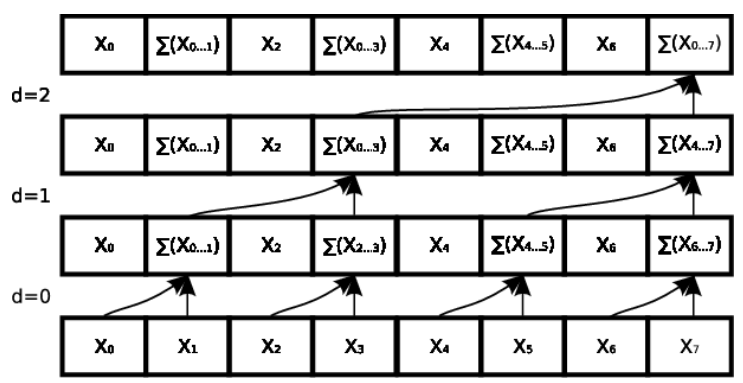

Fig. 3. Up-sweep phase

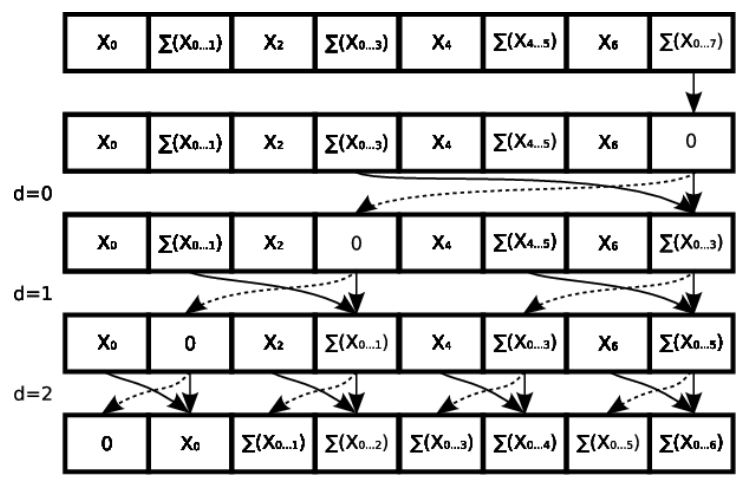

Fig. 4. Down-sweep phase 
2. Except $d=0$ in the up-sweep and $d=$ maxLevel in the down-sweep phase, only a fraction of workitems are active.

We suggest a solution for both problems, by using static workgroups. Figure 5 shows our solution to the first problem. As you can see, the whole computation is done with one kernel call. We subdivide the input array into as many blocks as we use static workgroups and have every static workgroups scan its part. We subdivide every block in smaller blocks and perform the scan identical to the one used by Harris et al. on these sub blocks. After the scan is complete, every static workgroups writes its result out to a SUM array. Note that the number of static workgroups does not depend on the input size of the array, which reduces the size of the SUM array. The SUM array is scanned by the last static workgroup to finish its part of the input array. We identify the last block by atomatically increment a shared counter in global memory. A global barrier is placed, so all other static workgroups wait until the work of the last workgroup is completed. The barrier is implemented following a wait $\rightarrow$ notify all concept. One counter is being used to identify the last block reaching the barrier. The last block notifies all other blocks by setting a flag. After the barrier, the final array is updated. We refer to this version solving problem 1 simply as $V 1$.

Our approach to solve problem 2 is to execute both phases in parallel. Figure 6 shows this in detail. We refer to this version as Scan $^{+}$. Executing both stages in parallel results in a higher workitem utilization in every step of the upand down-sweep phase and thereby decreases the time it takes to compute both phases. In the first step one workitem has to execute an instruction of both the up- and down-sweep phase, but in every other step there is up to one task for each workitem. Figure 7 shows the performance of both V1 and $\mathrm{Scan}^{+}$in comparison with Apple's implementation. Scan ${ }^{+}$outperforms Apple's implementation in

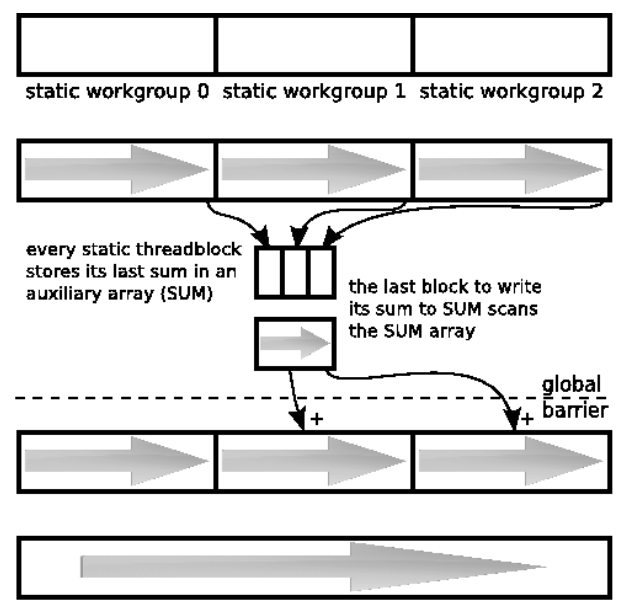

Fig. 5. Global overview of the $\mathrm{Scan}^{+}$algorithm 


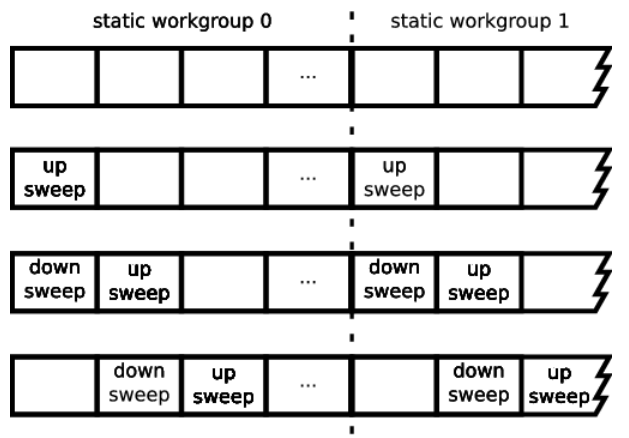

Fig. 6. Overlapping both scan phases

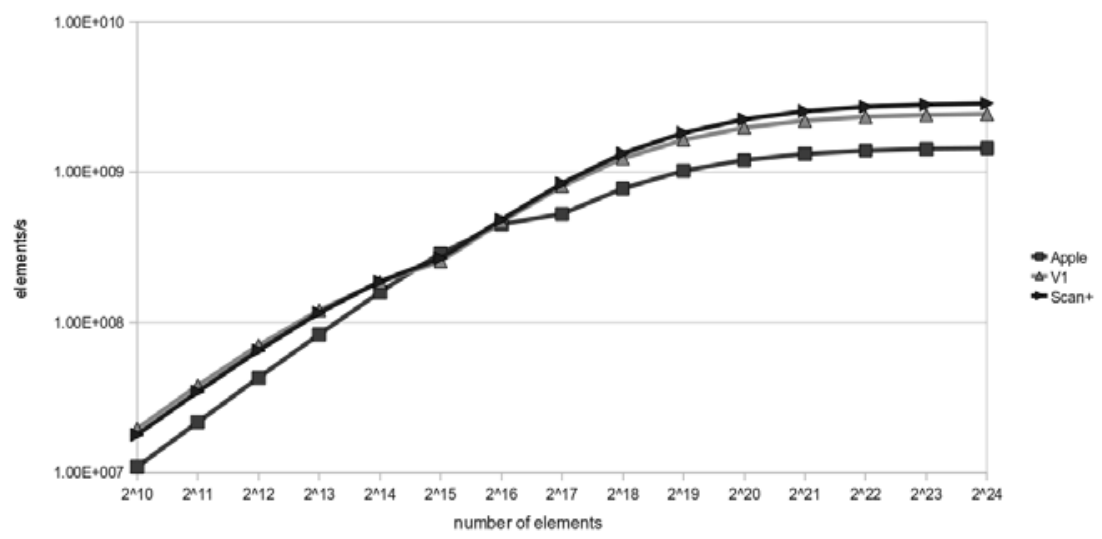

Fig. 7. GTX 280 scan performance

almost all cases. All three version use shared memory padding to prevent shared memory bank conflicts and unrolled loops to increase performance.

Our implementation is mostly limited by register pressure. If hardware would provide us with more registers, we could e.g. implement global memory prefetching as it has been suggested by [4] to increase our performance further. The performance is also limited by the strict synchronization primitives made available by OpenCL. Synchronization within a workgroup is only possible with a classic barrier, meaning every workitem of the workgroup has to reach the same barrier. However, NVIDIA hardware allows for finer grained synchronization [6] with barriers of which only a subset of workitem of a workgroup have to participate.

\section{Related Work}

Our implementation of the scan algorithm is based on Harris et al.'s work [5] and gains from their optimizations. Xiao et al. 8] studied fast barrier synchronization 
between threadblocks. Their fastest implementation differs to our solution by using arrays of counters. Stuart et al. 7] have implemented a MPI like interface for cooperate work of multiple MPEs. In previous work [3], we have showed that sharing data between work groups in OpenCL is also beneficial for the Cell Broadband Engine.

\section{Conclusion}

In this paper, we demonstrated that manual work distribution on GPUs increased flexibility in algorithm design, which can result in increased performance. We introduced the notion of static workgroups to effectively allow manual work distribution and evaluate its overhead compared to the native work distribution based on matrix multiplication. As a result there is hardly any performance difference between automatic and manual work distribution. Manual work distribution however allows for more flexibility in algorithm design, including global synchronization. We used the parallel execution of two tasks in one static workgroup and global synchronization to improve the scan performance by almost a factor of two. Future work will identify the strength and weaknesses of manual work distribution in different algorithms and different hardware architectures.

\section{References}

1. Apple Inc. OpenCL Parallel Prefix Sum (aka Scan) Example Version 1.5. (2010), http://developer .apple.com/mac/library/samplecode/OpenCL_Parallel_ Prefix_Sum_Example/Introduction/Intro.html

2. Blelloch, G.E.: Scans as primitive parallel operations. IEEE Trans. Computers 38(11), 1526-1538 (1989)

3. Breitbart, J., Fohry, C.: OpenCL - an effective programming model for data parallel computations at the Cell Broadband Engine. In: IEEE Int. Parallel and Distributed Processing Symposium (2010) (to appear)

4. Kirk, D., Hwu, W.-m.: Programming Massively Parallel Processors: A Hands-on Approach, 1st edn. Morgan Kaufmann, San Francisco (February 2010)

5. Harris, M., Sengupta, S., Owens, J.D.: Parallel prefix sum (scan) with CUDA. In: Nguyen, H. (ed.) GPU Gems 3, ch. 39, pp. 851-876. Addison Wesley, Reading (August 2007)

6. NVIDIA Corporation. PTX: Parallel Thread Execution ISA Version 2.0 (2010)

7. Stuart, J.A., Owens, J.D.: Message passing on data-parallel architectures. In: Stuart, J.A., Owens, J.D. (eds.) Proceedings of the 23rd IEEE International Parallel and Distributed Processing Symposium (May 2009)

8. Xiao, S., Feng, W.-c.: Inter-Block GPU Communication via Fast Barrier Synchronization. In: Proceedings of the 24th IEEE International Parallel and Distributed Processing Symposium (IPDPS), Atlanta, Georgia, USA (April 2010) 